\title{
COMPLETE METRICS WITH NONPOSITIVE CURVATURE ON THE DISK
}

\author{
DUONG MINH DUC
}

(Communicated by Barbara L. Keyfitz)

ABSTRACT. We consider complete metrics on the unit disk in $\mathbb{R}^{2}$ that are equivalent and conformal to the Poincaré metric.

\section{INTRODUCTION}

Let $D=\left\{x \in \mathbb{R}^{2}:|x|<1\right\}$ and $\widetilde{K}$ be a real function on $D$. In [6], Kazdan asked when $\widetilde{K}$ was the Gauss curvature of a complete metric conformal to the Poincaré metric on $D$. In $[1,2,5]$ we have the "Yes" answer under the following condition:

(0.1) There exist two negative constants $a$ and $b$ such that in a neighborhood of the boundary $\partial D$ of $D$

$$
a \leq \widetilde{K} \leq b<0 .
$$

We also have the "No" answer in [2] if $\widetilde{K}$ is nonnegative in a neighborhood of $\partial D$. In this paper we replace $(0.1)$ by $L^{p}$-conditions weaker than the old one. It is natural to use $L^{p}$-conditions because we define the length of curves by integration.

Our method is as follows: Let $K_{0}$ be a nonpositive Hölder-continuous function on $D$, which is the Gauss curvature of a metric $\rho=e^{u_{0}}\left(d x^{2}+d y^{2}\right)$ such that $\rho$ is equivalent to the Poincare metric. Consider the perturbations $\widetilde{K}=\left(K+K_{0}\right)$ and $v=u+u_{0}$ of $K_{0}$ and $u_{0}$, respectively. We try to find conditions on $K$ such that there is a bounded solution of the equation

$$
\Delta u=K_{0} e^{2 u_{0}}=\left(K+K_{0}\right) e^{2 u_{0}} e^{2 u} \text { in } D .
$$

(Note that $\Delta u_{0}=-K_{0} e^{2 u_{0}}$.) When $K_{0}=-1, e^{2 u_{0}}=4\left(1-|x|^{2}\right)^{-2}$, and $(0.2)$ becomes

$$
\Delta u=-\frac{4}{\left(1-|x|^{2}\right)^{2}}-\frac{4(K-1) e^{2 u}}{\left(1-|x|^{2}\right)^{2}} \text { in } D .
$$

Our results are the following theorems.

Received by the editors March 22, 1990 and, in revised form, June 28, 1990.

1980 Mathematics Subject Classification (1985 Revision). Primary 35J20, 53A30.

Key words and phrases. Conformal complete metric, Gauss curvature, nonlinear elliptic equation. 
Theorem 1. Let $\widetilde{K}$ be a nonpositive Hölder-continuous function on $D$ and $K_{0}$ a nonpositive Hölder-continuous function on $D$, which is the Gauss curvature of a metric conformal and equivalent to the Poincare metric. Then

(i) There is a solution $u$ in $W_{0}^{1,2}(D) \cap C^{2}(D)$ of $(0.2)$, if

$$
\int_{D} \frac{\left|\tilde{K}-K_{0}\right|^{2}}{(1-|x|)^{2}} d x<\infty .
$$

(ii) Moreover, this solution is bounded if there are positive real numbers $p$ and $\varepsilon$ such that $p>1, \frac{p}{p-1}(1-\varepsilon)<1$, and

$$
\int_{D} \frac{\left|\widetilde{K}-K_{0}\right|^{p(1+\varepsilon)}}{(1-|x|)^{p(1+\varepsilon)^{2}}} d x<\infty .
$$

Theorem 2. Let $\widetilde{K}$ be a nonpositive Hölder-continuous function on $D$ and $K_{0}$ $a$ Hölder-continuous function from $D$ into $[a, b], a \leq b<0$. Then the conclusions of Theorem 1 hold.

Remark. Here $\widetilde{K}$ may not satisfy (0.1) for any $a$ and $b$. Some special examples of $\widetilde{K}$ considered in the theorems above have been given in [2].

In $\S \S 1$ and 2 we prove the theorems for the case $K_{0}=-1$, and we consider general cases in the last section.

\section{EXISTENCE}

Denote by $X$ the family of measurable functions $u$ such that

$$
\|u\|_{*}=\int_{D} \frac{u^{2}}{(1-|x|)^{2}} d x<\infty .
$$

Let $r \in(0,1)$ and $D_{r}=\left\{x \in \mathbb{R}^{2}:|x|<r\right\}$. We have the following lemma.

Lemma 1.1. Let $K \in X, r \in(0,1)$, and $v \in W_{0}^{1,2}\left(D_{r}\right)$. Then there exists a unique solution $u$ in $W^{2,2}\left(D_{r}\right)$ of the equation

$$
\Delta u=-\frac{4}{\left(1-|x|^{2}\right)^{2}}-\frac{4(K-1) e^{2 v}}{\left(1-|x|^{2}\right)^{2}} \text { in } D_{r} .
$$

Proof. Using the Trudinger imbedding theorem $[3,7,9]$, we see that the functions in the right-hand side are in $L^{2}\left(D_{r}\right)$. Thus by [4, Theorem 8.9, p. 185] we get the lemma.

Fix a $K$ in $X$. By Lemma 1.1, the following mapping is well defined:

$$
\begin{gathered}
F: W_{0}^{1,2}\left(D_{r}\right) \mapsto W_{0}^{2,2}\left(D_{r}\right) \\
F(v)=\Delta^{-1}\left(-\frac{4}{\left(1-|x|^{2}\right)^{2}}-\frac{4(K-1) e^{2 u}}{\left(1-|x|^{2}\right)^{2}}\right) .
\end{gathered}
$$

We have the following lemma. 
Lemma 1.2. Let $r \in(0,1)$ and $K \in X$. Assume $K-1 \leq 0$. Then there exists a solution $v_{r}$ in $W_{0}^{1,2}\left(D_{r}\right)$ of the equation

$$
\Delta v=-\frac{4}{\left(1-|x|^{2}\right)^{2}}-\frac{4(K-1) e^{2 v}}{\left(1-|x|^{2}\right)^{2}} \text { in } D_{r} .
$$

Moreover,

$$
\int_{D_{r}}\left|\nabla v_{r}\right|^{2} d x \leq 8 C\|K\|_{*}^{2},
$$

where $C$ is the constant in the following Hardy inequality:

$$
\int_{D} \frac{|u|^{2}}{(1-|x|)^{2}} d x \leq C \int_{D}|\nabla u|^{2} d x \quad \forall u \in W_{0}^{1,2}(D) .
$$

Proof. By [4, Lemma 9.17, p. 242] and the Sobolev imbedding theorem, we see that $F$ is a compact continuous mapping from $W_{0}^{1,2}\left(D_{r}\right)$ into itself. Put

$$
A=\left\{w \in W_{0}^{1,2}\left(D_{r}\right): w=t F w \text { for some } t \in(0,1)\right\} .
$$

We shall prove that $A$ is bounded in $W_{0}^{1,2}\left(D_{r}\right)$. Indeed, let $t \in(0,1)$ and $w \in W_{0}^{1,2}\left(D_{r}\right)$ such that

$$
\Delta w=-\frac{4 t}{\left(1-|x|^{2}\right)^{2}}-\frac{4 t(K-1) e^{2 w}}{\left(1-|x|^{2}\right)^{2}} \text { in } D_{r}
$$

Put $w^{+}=\max \{0, w\}$ and $w^{-}=\max \{0,-w\}$. Multiplying both sides of (1.5) by $-w$ and integrating them on $D_{r}$, we get

$$
\begin{aligned}
& \int_{D_{r}}|\nabla w|^{2} d x=4 t \int_{D_{r}}\left\{\frac{w}{\left(1-|x|^{2}\right)^{2}}+\frac{(K-1) w e^{2 w}}{\left(1-|x|^{2}\right)^{2}}\right\} d x, \\
& \quad \leq 4 \int_{D_{r}} \frac{1}{\left(1-|x|^{2}\right)^{2}}\left\{w^{+}+(K-1) w^{+} e^{2 w^{+}}-w^{-}-(K-1) w^{-} e^{-2 w^{-}}\right\} d x .
\end{aligned}
$$

Since $K-1 \leq 0$, we have

(1.7) $(K-1) w^{+} e^{2 w^{+}} \leq(K-1) w^{+}$and $-(K-1) w^{-} e^{-2 w^{-}} \leq-(K-1) w^{-}$.

By (1.6) and (1.7), we obtain

$$
\begin{aligned}
\int_{D_{r}}|\nabla w|^{2} d x & \leq 4 \int_{D_{r}} \frac{K w}{\left(1-|x|^{2}\right)^{2}} d x \\
& \leq 4 C \int_{D_{r}} \frac{|K|^{2}}{(1-|x|)^{2}} d x+\frac{1}{2 C} \int_{D_{r}} \frac{|w|^{2}}{(1-|x|)^{2}} d x .
\end{aligned}
$$

By (1.4) and (1.8), we see that

$$
\int_{D_{r}}|\nabla u|^{2} d x \leq 8 C\|K\|_{*}^{2}
$$


Thus, $A$ is bounded in $W_{0}^{1,2}\left(D_{r}\right)$. Applying the Leray-Schauder principle [10, p. 245], we get a fixed point $v_{r}$ of $F$, which is a solution of (1.2) and satisfies (1.3).

The proof of (i) of Theorem 1 for $K_{0}=-1$. Let $\left\{r_{m}\right\}$ be a sequence converging to 1 in $(0,1)$ and $u \in W_{0}^{1,2}(D)$ such that $\left\{\left.u_{m}\right|_{D_{r}}\right\}$ weakly converges to $\left.u\right|_{D_{r}}$ in $W_{0}^{1,2}\left(D_{r}\right)$ for any $r$ in $(0,1)$, where $u_{m}=v_{r_{m}}$ is defined as in Lemma 1.2. Using [3, Corollary 1.3] we see that $u$ is a weak solution of $W_{0}^{1,2}(D)$ and satisfies (1.3).

\section{BOUNDEDNESS}

First we need the following lemma.

Lemma 2.1. Let $\Omega$ be a bounded domain in $\mathbb{R}^{n}$ with piecewise-smooth boundary $\partial \Omega$. Let $g$ be a nonnegative measurable function on $\Omega$ and $h$ be in $L^{p}(\Omega)$ for some $p>n / 2$. Let $v$ be a nonnegative weak solution in $W_{0}^{1,2}(\Omega) \cap L^{\infty}(\partial \Omega)$ of the equation

$$
\Delta v=g+h \text { in } \Omega \text {. }
$$

Then $\|v\|_{\infty}$ is finite and bounded above by a constant depending only on $p$, $\|h\|_{p}$, and $\|v\|_{1}$.

Proof. Let $k$ be an arbitrary positive real number and $\zeta$ an arbitrary smooth nonnegative function of compact support in $\Omega$ whose values lie between 0 and 1. Put

$$
\begin{aligned}
\eta(x) & =\zeta^{2}(x) \max \{v(x)-k ; 0\}, \\
A_{k} & =\{x \in \Omega: v(x)>k\} .
\end{aligned}
$$

By some simple calculations, we get

$$
\int_{A_{k}}\left\{|\nabla v|^{2} \zeta^{2}+\sum_{j=1}^{m} 2 \frac{\partial v}{\partial x_{j}}(v-k) \cdot \zeta \frac{\partial \zeta}{\partial x_{j}}+(g+h)(v-k) \zeta^{2}\right\} d x=0 .
$$

Thus,

$$
\int_{A_{k}}|\nabla v|^{2} \zeta^{2} d x \leq \int_{A_{k}}\left\{\frac{1}{2}|\nabla v|^{2} \zeta^{2}+2|\nabla \zeta|^{2}(v-k)^{2}+|h|(v-k) \zeta^{2}\right\} d x
$$

Now, following the proof of [8, Theorem 13.1, pp. 197-199], we get the lemma.

In order to prove the boundedness of the solution $u$ in $\S 1$, it is sufficient to show that there is a constant $M$ such that $\left\|v_{r}\right\|_{\infty} \leq M$ for every $r$ in $(0,1)$, where $v_{r}$ is as in Lemma 1.2. Indeed, let $r$ be in $(0,1), w_{r}=v_{r}^{+}$, and $\Omega$ be an open subset of $\left\{x \in D_{r}: v_{r}>0\right\}$ with piecewise smooth boundary $\partial \Omega$. Since $v_{r}$ is in $W_{0}^{2,2}\left(D_{r}\right)$, by the Sobolev imbedding theorem $v_{r}$ is in $L^{\infty}\left(D_{r}\right)$. Note that $w_{r}$ is a nonnegative weak solution in $W^{1,2}(\Omega)$ of

$$
\Delta w_{r}=4 \frac{e^{2 w_{r}}-1}{\left(1-|x|^{2}\right)^{2}}-\frac{4 K e^{2 w_{r}}}{\left(1-|x|^{2}\right)^{2}} .
$$


On the other hand, we have

$$
\left\|v_{r}\right\|_{1} \leq\left\|v_{r}\right\|_{2} \leq C\|\nabla u\|_{2} \leq 8 C^{2}\|K\|_{*}
$$

and

$$
\begin{aligned}
\int_{D}\left|\frac{K e^{2 v_{r}}}{\left(1-|x|^{2}\right)^{2}}\right|^{1+\varepsilon} d x & \leq \int_{D} \frac{|K|^{1+\varepsilon} e^{2(1+\varepsilon) v_{r}}}{(1-|x|)^{(1+\varepsilon)^{2}+\left(1-\varepsilon^{2}\right)}} \\
\leq & \left\{\int_{D} \frac{|K|^{p(1+\varepsilon)}}{(1-|x|)^{p(1+\varepsilon)^{2}}} d x\right\}^{1 / p} \\
& \times\left\{\int_{D} \frac{e^{2 p(1+\varepsilon) v_{r} /(p-1)}}{(1-|x|)^{p\left(1-\varepsilon^{2}\right) /(p-1)}} d x\right\}^{(p-1) / p} .
\end{aligned}
$$

Using Theorem 1.3 in [3], we can estimate the last integral in (2.3) by $\left\|\nabla v_{r}\right\|_{2}$, then by $\|K\|_{*}$. Therefore, by applying Lemma 2.1 , we see that $\left\{\left\|w_{r}\right\|_{\infty}\right\}_{r}$ is bounded.

Put $z_{r}=v_{r}^{-}$. Then $z_{r}$ is a nonnegative weak solution of

$$
\Delta z_{r}=4 \frac{1-e^{-2 z_{r}}}{\left(1-|x|^{2}\right)^{2}}+\frac{4 K e^{-2 z_{r}}}{\left(1-|x|^{2}\right)^{2}} \quad \text { in }\left\{x \in D: v_{r}<0\right\} \text {. }
$$

Arguing as above, we see that $\left\{\left\|z_{r}\right\|_{\infty}\right\}_{r}$ is bounded. Therefore $\left\{\left\|v_{r}\right\|_{\infty}\right\}_{r}$ is bounded and $\|u\|_{\infty}$ is finite.

\section{General cases}

Let $K_{0}$ be as in Theorem 1. Then there is a $C^{2}$-function $u_{0}$ on $D$ and two positive constants such that

$$
A e^{2 u_{0}} \leq 4\left(1-|x|^{2}\right)^{-2} \leq B e^{2 u_{0}}
$$

and

$$
\Delta u_{0}=-K_{0} e^{2 u_{0}}
$$

Let $K \in X$ and $v=u_{0}+u$. Then we have

$$
\Delta v+\left(K+K_{0}\right) e^{2 v}=0 \Leftrightarrow \Delta u=K_{0} e^{2 u_{0}}-\left(K+K_{0}\right) e^{2 u_{0}} e^{2 u} .
$$

Assume that $\widetilde{K}=K+K_{0} \leq 0$. Then, by (3.1), (3.3) is similar to (1.1). Therefore, by a similar procedure we can prove Theorem 1 for the case $K_{0} \neq-1$.

Now let $a<b<0$ and $K_{0}$ be a $C^{2}$-function from $D$ into $[a, b]$. By results in [1, 2], $K_{0}$ satisfies the conditions of Theorem 1. Therefore we get Theorem 2.

\section{ACKNOWLEDGMENTS}

The author would like to thank Professor Abdus Salam, the International Atomic Energy Agency, and UNESCO for their hospitality at ICTP. He also would like to thank Professor Alberto Verjovsky for warm and successful discussions and the referee for reference [5]. 


\section{REFERENCES}

1. P. Aviles and R. McOwen, Conformal deformations of complete manifolds with negative curvature, J. Differential Geom. 21 (1985), 269-281.

2. J. Bland and M. Kalka, Complete metrics conformal to the hyperbolic disc, Proc. Amer. Math. Soc. 97 (1986), 128-132.

3. D. M. Duc, Nonlinear singular elliptic equations, J. London Math. Soc. (2) (to appear).

4. D. Gilbarg and N. S. Trudinger, Elliptic partial differential equations of second order, Springer-Verlag, Berlin, 1983.

5. J. A. Goldstein and C. Y. Lin, Highly degenerate parabolic boundary value problems, Differential Integral Equations 2 (1989), 216-227.

6. J. L. Kazdan, Prescribing the curvature of a Riemannian manifold, Regional Conf. Ser. in Math., no. 57, Amer. Math. Soc., Providence, RI, 1985.

7. J. L. Kazdan and F. Warner, Curvature functions for compact 2-manifolds, Ann. of Math. (2) 99 (1974), 14-47.

8. O. A. Ladyzhenskya and N. N. Ural''tseva, Linear and quasilinear elliptic equations, Academic Press, New York, 1968.

9. N. S. Trudinger, On embeddings into Orlicz spaces and some applications, J. Math. Mech. 17 (1967), 473-483.

10. E. Zeidler, Nonlinear functional analysis and its applications, vol. I, Springer-Verlag, Berlin, 1985.

International Centre for Theoretical Physics, Trieste, Italy

Current address: Department of Mathematics, University of Hochiminh City, Hochiminh City, Vietnam 